\title{
ChemComm
}

\section{Streptavidin binding as a model to characterize thiol-ene chemistry-based polyamine surfaces \\ for reversible photonic protein biosensing $\dagger$}

Cite this: Chem. Commun., 2014, 50,2424

Received 12th November 2013, Accepted 25th December 2013

DOI: $10.1039 / \mathrm{c3cc} 48640 \mathrm{k}$

www.rsc.org/chemcomm

\author{
Eva Melnik, ${ }^{\text {a }}$ Paul Muellner, ${ }^{a}$ Ole Bethge, ${ }^{b}$ Emmerich Bertagnolli, ${ }^{b}$ \\ Rainer Hainberger ${ }^{a}$ and Michael Laemmerhofer ${ }^{c}$
}

\begin{abstract}
Biotin- and iminobiotin-bonded surfaces obtained by thiol-ene chemistry and subsequent modification with polyamines were characterized with respect to streptavidin-binding capacity and reversibility for photonic biosensing using X-ray photoelectron spectroscopy and Mach-Zehnder-interferometric sensors. The streptavidin-iminobiotin system was exploited for reversible multilayer deposition and determination of affinity constants on each layer.
\end{abstract}

Kinetic studies of receptor-protein interactions play a pivotal role in medical research and drug development. Comprehensive analyses of kinetic parameters necessitate measurements for a large variety of receptors and proteins at different levels of concentrations. In order to facilitate these analyses, a surface modification approach is desirable that provides high binding capacity and offers the possibility of repeated measurements on a single regenerative sensor array.

One promising strategy to obtain high binding capacities is the use of a multifunctional polymer grafting layer that increases the density of surface functionalities (e.g. amines) and allows immobilizing large amounts of receptors. The surface grafting of polymers can be performed by non-covalent adsorption. ${ }^{1}$ However, this causes a collapse of the $3 \mathrm{D}$ polymer shape. ${ }^{2}$ The goal of this work is to extend flat planar 2D surface modifications to voluminous 3D surface layers, which are not broadly utilized although they may be promising strategies to enhance saturation capacities. For biosensing, the globular-shaped dendrimers $^{2}$ and linear-shaped brush polymers ${ }^{3}$ are of interest to gain well orientated surface receptors or to form voluminous 3D surface layers, respectively. During the surface grafting process, the primary shape of the polymer has to be preserved

\footnotetext{
${ }^{a}$ AIT Austrian Institute of Technology GmbH, Health \& Environment Department, Molecular Diagnostics, 1220 Vienna, Austria.E-mail:Eva.Melnik@ait.ac.at; Web: www.ait.ac.at

${ }^{b}$ Institute for Solid-State Electronics, Vienna University of Technology, Floragasse 7 , 1040 Vienna, Austria

${ }^{c}$ University of Tübingen, Institute of Pharmaceutical Sciences, Auf der Morgenstelle 8, Tübingen, Germany

$\dagger$ Electronic supplementary information (ESI) available. See DOI: 10.1039/ c3cc48640k
}

and, therefore, covalent immobilization strategies are more promising than non-covalent adsorption methods. ${ }^{2}$ For the covalent surface grafting, we investigated thiol-ene coupling-based surface modifications with subsequent derivatization of the resulting carboxylic groups using selected polyamine-type polymers. The thiol-ene coupling was chosen owing to its mild reaction conditions, quantitative yields, and its feasibility in the presence of oxygen. ${ }^{4}$ In addition, it allows a spatially controlled surface modification employing optical lithography or laser writing with feature sizes down to the micron range. ${ }^{5}$ In this study, we selected polyamidoamines (PAMAM) G1 and G5, as well as polyethyleneimine (PEI, $750 \mathrm{kDa}$ ), to compare dendrimers that have significantly different hydrodynamic diameters with a more cost-effective brush polymer. These polyamines were systematically characterized with respect to their chemical composition using X-ray photoelectron spectroscopy (XPS) and with respect to their binding capacity and reversibility using two streptavidinbinding models based on biotin and iminobiotin (see Fig. 1). On the basis of these results, we devised a novel reversible multilayer deposition concept which allows repeated determination of the association constant either at each layer or as an enhanced cumulative signal.

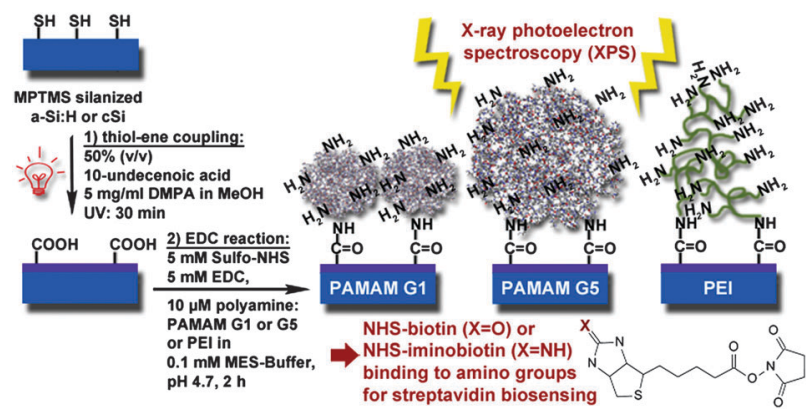

Fig. 1 Surface modification steps of crystalline silicon (cSi) and amorphous hydrogenated silicon (a-Si:H): (1) activation by thiol-ene coupling; (2) EDC reaction for the covalent binding of PAMAM G1, G5 and PEI. The resultant sensor surfaces were characterized by XPS and streptavidin biosensing. Total sensor preparation time is $\sim 6 \mathrm{~h}$. 
As a testing platform, we employed silicon-based photonic sensors, which have been demonstrated to be an attractive means to perform kinetic studies. ${ }^{6}$ The sensing principle relies on optical wave guiding due to total internal reflection. A part of the optical field of the guided light - the so called evanescent field - penetrates into the surrounding media and decays exponentially. Typical penetration depths of the evanescent field are in the range of $60-150 \mathrm{~nm}$ for light with a wavelength of $1.3 \mu \mathrm{m}$. Binding of the target molecule to the functionalized waveguide surface locally changes the refractive index, which induces a change of the phase velocity of the guided light. We use amorphous hydrogenated silicon (a-Si:H) based Mach-Zehnder interferometric (MZI) sensors (see Fig. S1, ESI†) to translate the change of the phase velocity into a modulation of the optical output power. From this sinusoidal optical output an accumulated phase shift $(\Delta \Phi)$ is calculated, which correlates with the amount of target molecules captured on the surface. The silicon-based photonic biosensors can be made of different materials (e.g. a-Si:H, crystalline silicon, silicon nitride). ${ }^{6}$ One prominent method for functionalizing these materials is surface modification using self-assembled functional silane layers. ${ }^{6}$ In this study, we performed this silanization using (3-mercaptopropyl)trimethoxysilane (MPTMS) on two specific types of silicon - hydrogenated amorphous silicon and undoped crystalline (100) silicon (cSi). The optimized silanization procedures for these substrates resulted in significantly higher surface densities of reactive sulfhydryl groups for a-Si:H (Table S1, ESI $\dagger$ ). This preliminary study emphasizes the need for a subsequent surface modification procedure that ensures equally high surface concentrations of functional groups irrespective of the substrate. We investigated whether thiol-ene coupling combined with the selected polyamines can provide an equalization of the functional group density on a-Si:H and cSi. To achieve the covalent binding of the selected multifunctional polymers, the stable MPTMS-derivatized silicon surfaces were activated by photo-induced thiol-ene coupling. This radical addition reaction between surface-bound thiol groups and 10-undecenoic acid (UDA) was first optimized on a-Si:H control

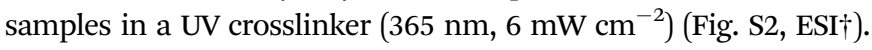
The optimized thiol-ene coupling protocol was then transferred to cSi surfaces. To evaluate the performance of this surface modification chemistry on a-Si:H and cSi, ethylene diamine (EDA) was bound to the surface carboxy groups and the resulting amino groups were labelled with NHS-DyLight. Fluorescence scans of these surfaces revealed a fluorescence intensity which is three times higher for a-Si:H surfaces than for cSi surfaces (Fig. S3, ESI $\dagger$ ).

In the next step, polyamines were covalently bound to the terminal carboxylic acid groups of the a-Si:H and cSi surfaces modified by thiol-ene coupling (see Fig. 1). To analyse the equalization effect of the multifunctional polymers, ethylene diamine was also bound to the modified surfaces and characterized as a representative for surface modifications using a small amine derivative. The characterization was performed by means of XPS (Mg K $\alpha, 1253.6 \mathrm{eV}$, take-off angle $\theta=0^{\circ}$ ). This surface analysis technique enables the determination of atomic composition, functional group concentration (e.g. of amino and thiol groups) and layer thickness to be carried out. These are important parameters for protein sensing employing surface grafted polymer layers. The evaluation of the atomic
Table 1 XPS ( $M g ~ K \alpha, 1253.6 \mathrm{eV}$, take-off angle $\theta=0^{\circ}$ ) data including the atomic composition, reactive primary amino group concentration and modification thickness $d$ of EDA, PAMAM G1 and G5, PEI surfaces

\begin{tabular}{|c|c|c|c|c|c|c|c|c|}
\hline \multirow{2}{*}{$\begin{array}{l}\text { Atomic } \\
\text { composition (\%) }\end{array}$} & \multicolumn{2}{|l|}{ EDA } & \multicolumn{2}{|c|}{ PAMAM G1 } & \multicolumn{2}{|c|}{ PAMAM G5 } & \multicolumn{2}{|l|}{ PEI } \\
\hline & a-Si:H & $\mathrm{cSi}$ & a-Si:H & $\mathrm{cSi}$ & a-Si:H & $\mathrm{cSi}$ & $\mathrm{a}-\mathrm{Si}: \mathrm{H}$ & $\mathrm{cSi}$ \\
\hline Si2p & 37.7 & 49.0 & 33.8 & 39.5 & 28.0 & 31.6 & 27.4 & 34.6 \\
\hline O1s & 37.0 & 35.8 & 32.3 & 32.1 & 31.3 & 32.0 & 26.4 & 30.7 \\
\hline C1s & 13.9 & 12.5 & 20.8 & 24.0 & 26.8 & 30.6 & 30.0 & 26.7 \\
\hline N1s & 11.5 & 2.7 & 13.1 & 4.3 & 14.0 & 5.8 & 16.2 & 8.0 \\
\hline $\mathrm{R}-\mathrm{NH}_{2}(\%)$ & 1.4 & 0.9 & 2.2 & 2.4 & 3.9 & 3.8 & 6.6 & 6.5 \\
\hline$d(\mathrm{~nm})$ & 0.46 & 0.33 & 0.91 & 1.09 & 1.37 & 1.79 & 1.23 & 1.93 \\
\hline
\end{tabular}

composition is summarized in Table 1 and reveals an increase of nitrogen (N1s) and carbon (C1s) in ascending order for EDA, G1, G5 and PEI surfaces. The peak deconvolution of the N1s signals allows the primary, secondary/tertiary amines, amides and protonated amines to be differentiated (Table S2, Fig. S5 and S6, ESI $\dagger$ ). While tertiary amines do not react in EDC coupling, and secondary amines need stronger activation, primary amines are reactive ${ }^{7}$ and thus are of particular importance for further modification with NHS-(imino)biotin. In accordance with the preliminary fluorescence measurements, the primary amino group concentrations $\left(\mathrm{R}-\mathrm{NH}_{2}\right.$ ) (see Table 1) were significantly higher on EDA-modified a-Si:H than on EDA-modified cSi. On the other hand, the controlled modification with a defined polyamine leads to an equalizing effect. Thus, the type of substrate no longer determines the surface concentration of reactive primary amino groups available for (imino)biotin coupling reaction. Furthermore, as a beneficial side effect, the concentration of $\mathrm{R}-\mathrm{NH}_{2}$ for G1, G5, and PEI surfaces increases in ascending order (see Table 1). For the analysis of the layer thickness, the attenuation length $\lambda$ of the photoelectrons was estimated from their kinetic energy $\left(E_{\mathrm{K}}\right)$ using the equation of Laibinis et al. ${ }^{8}$ which has the form $\lambda\left(E_{\mathrm{K}}\right)=9+0.022 E_{\mathrm{K}}$. With the estimated attenuation length $(\lambda=34.3 \AA)$ derived from the photoelectron kinetic energy $\left(E_{\mathrm{K}}\right)$ of the Si2p peak, the polymer layer thickness $d$ was calculated using a modified Lambert-Beer equation: ${ }^{9}$

$$
d=-\lambda \ln \left(I / I_{0}\right) \cos \theta,
$$

where $I$ and $I_{0}$ are the Si2p peak intensities of the functionalized and non-modified surfaces, respectively, measured for a-Si:H and cSi. From the XPS measurement results summarized in Table 1, it can be concluded that PAMAM G5 and PEI provide the best conditions for protein sensing in terms of modification thickness and primary amino group concentration.

To analyse the polyamine surfaces with respect to binding capacity, reversibility, and kinetic behaviour, measurements on a-Si:H MZI sensors were performed using a streptavidin-binding model based on biotin and iminobiotin. The biotin-streptavidin interaction is one of the strongest known in nature with a dissociation constant of $K_{\mathrm{D}}=10^{-15} \mathrm{M}$, which under mild conditions prevents reversibility of binding. ${ }^{10}$ The analogue iminobiotin has a weaker and $\mathrm{pH}$-dependent association to streptavidin due to its guanidinium moiety in the bicyclic ring replacing the urea functionality. ${ }^{11}$ Owing to these binding characteristics, biotin was used to analyse the protein binding capacity, and iminobiotin to perform 
reversible measurements. For this purpose, the polyamines were (imino)biotinylated using NHS derivatives (S6, ESI $\dagger$ ). Before the measurements, the (imino)biotinylated sensor surfaces were rinsed with bovine serum albumin (100 $\mathrm{g} \mathrm{ml}^{-1}$ in $15 \mathrm{mM}$ PBS, $\left.10 \mathrm{~min}\right)$ to block low energy binding sites and suppress non-specific interactions on the surfaces. The MZI sensor measurements were performed as fast screening experiments at high streptavidin concentration (1666 $\mathrm{pmol} \mathrm{ml}^{-1}$ ) to obtain maximum signal output in reasonable time. Physiological conditions (PBS $15 \mathrm{mM}, \mathrm{pH} 7.5$ ) were used to comply with biosensing requirements. The biotinylated sensors show an increased response from $\Delta \Phi=2.1 \times 2 \pi$ for EDA, $3.7 \times 2 \pi$ for PAMAM G1 and $5.3 \times 2 \pi$ for G5, to $6.4 \times 2 \pi$ for PEI (Fig. 2). After about $1500 \mathrm{~s}$ the streptavidin solution was replaced by a plain buffer solution. As expected, no dissociation of streptavidin occurs on biotinylated surfaces. In contrast, reversible binding of streptavidin was possible on the iminobiotinylated polyaminemodified MZI sensors. Measurements using these sensors were performed by a sequence of four consecutive binding (with $1666 \mathrm{pmol} \mathrm{ml}^{-1}$ streptavidin solution), dissociation (with buffer rinsing) and regeneration experiments. Complete regeneration of the surface was realized by rinsing with $3.5 \mathrm{mM}$ hydrochloric acid. The sensor response also increased on these iminobiotinylated surfaces from $\Delta \Phi=1.54 \pm 0.059 \times 2 \pi$ for PAMAM G1 and $1.78 \pm 0.41 \times 2 \pi$ for G5 to $1.94 \pm 0.19 \times 2 \pi$ for PEI (see Fig. 2). In comparison with the biotin surfaces the sensor responses are lower because of a lower surface coverage with active imino-biotin functionalities, which is probably a result of the specific chemistry with the protected guanidine group of iminobiotin and its cleavage (S6, ESI $\dagger$ ). From the standard deviations $(n=4)$ it can be seen that the repeatability of the monitored phase shifts associated with the binding event at surface saturation was satisfactory for G1 and PEI but showed higher fluctuations for G5. The streptavidin-iminobiotin association constants were calculated for a better understanding of the protein-binding behaviour on these surfaces (Table S4, ESI $\dagger$ ). The association constants for iminobiotinylated surfaces increase in ascending order for G1 $\left(K_{\mathrm{A}}=4.7 \times 10^{5} \mathrm{M}^{-1}\right)$, G5 $\left(K_{\mathrm{A}}=9.3 \times 10^{5} \mathrm{M}^{-1}\right)$, and PEI $\left(K_{\mathrm{A}}=1.9 \times 10^{6} \mathrm{M}^{-1}\right)$. The value for PEI is nearly one order of magnitude higher, which clearly reveals higher binding affinities and higher binding capacities. In addition, three freshly prepared PEI-based iminobiotinylated MZI sensors show an average response of $\Delta \Phi=1.69 \pm 0.07 \times 2 \pi$, which indicates good reproducibility of the surface modification.

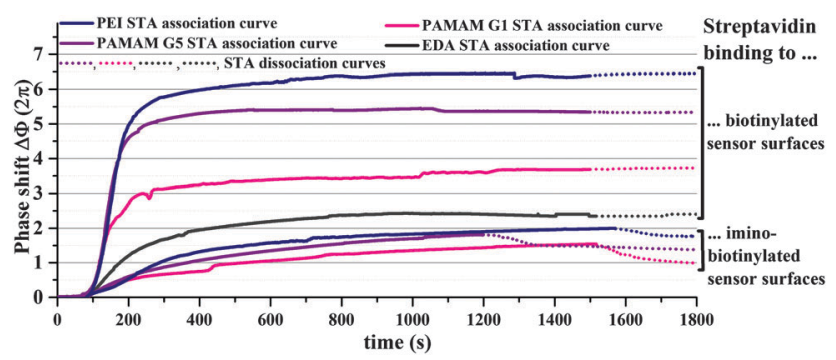

Fig. 2 Comparison of sensor responses during the measurement of 1666 pmol $\mathrm{ml}^{-1}$ streptavidin (STA) on biotinylated or iminobiotinylated EDA, PAMAM G1, G5 and PEI modified MZI sensors.
These comparative streptavidin binding experiments on the different (imino)biotinylated polyamine-modified MZI sensors confirm the results of XPS analysis and clearly emphasize the advantages of the surface chemistry involving PEI modifications. On the basis of these results, the PEI-based iminobiotin modification was chosen to examine the effect of multilayer deposition of alternating streptavidin (STA)-iminobiotinylated PEI chain (PEI-IB) coatings. The STA-PEI-IB bilayer stacks were deposited at different streptavidin concentrations (166-1666 pmol $\mathrm{ml}^{-1}$ ) and constant PEI-IB concentration $\left(50 \mu \mathrm{g} \mathrm{ml}^{-1}\right)$ (PEI-IB synthesis $\left.\mathrm{S} 7, \mathrm{ESI} \dagger\right)$. Five bilayer stacks were deposited at each streptavidin concentration. Finally, the sensor was regenerated by rinsing with $3.5 \mathrm{mM}$ hydrochloric acid $(5 \mathrm{~min})$. The signal increases linearly from the first to the fifth bilayer (Fig. S8, $\mathrm{ESI} \dagger$ ), which indicates a concentration dependent growth behaviour and provides an enhancement of the sensor response. To determine the association constant, the sensor response obtained at a certain number of bilayer stacks was plotted against the different streptavidin concentrations (see Fig. 3A). The association constants were then calculated using the Langmuir isotherm:

$$
S=S_{\max } C /\left(\left(1 / K_{\mathrm{A}}\right)+C\right)
$$

where $S$ is the actual and $S_{\max }$ is the maximum signal at infinitely high concentrations in one particular bilayer stack, $C$ is the streptavidin concentration in the feed solution, and $K_{\mathrm{A}}$ is the association constant. The determination of the association constant shows that the streptavidin affinity to the imino-biotinylated surface decreases from the first $\left(K_{\mathrm{A}}=2.2 \times 10^{6} \mathrm{M}^{-1}\right)$ to the third $\left(K_{\mathrm{A}}=5.7 \times 10^{5} \mathrm{M}^{-1}\right)$ bilayer stack and then stays almost constant (Fig. 3B) (Table S5, ESI $\dagger$ ). This behaviour indicates that the STA-PEI-IB bilayer stack deposition leads to a change of the surface-binding properties (e.g. sterical arrangement or concentration of iminobiotin) until a self-assembled equilibrium is obtained. In the literature, streptavidin-iminobiotin association constants were determined in buffer solutions ( $\mathrm{pH} 7.5)$ by Green ${ }^{12}$ and Raphael et al. ${ }^{13}$ with $K_{\mathrm{A}}$ values of $1.26 \times 10^{6} \mathrm{M}^{-1}$ and $1.58 \times 10^{5} \mathrm{M}^{-1}$, respectively.

In conclusion, the characterization of the thiol-ene chemistry based multifunctional polyamine surfaces clearly documented the advantages of PEI modifications for protein sensing. The PEI polymer is not as highly ordered as the PAMAM G5 dendrimer, but leads to higher surface amino group concentrations and
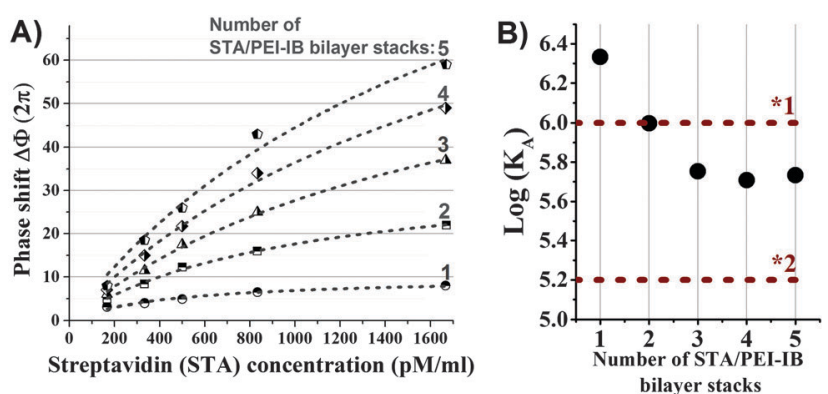

Fig. 3 (A) Sensor signal plotted against the different streptavidin (STA) concentrations, shown for the number of bilayer stacks. (B) Logarithmic value of association constant $K_{\mathrm{A}}$ plotted against the number of bilayer stacks. The literature values of Green $\left({ }^{*} 1\right)^{12}$ and Raphael et al. $\left({ }^{*} 2\right)^{13}$ were determined for the STA-iminobiotin interaction in buffer solution. 
protein loading capacities. Furthermore, the properties of iminobiotinylated PEI surfaces can be utilized for reversible biosensing, as shown in this study. The reversible streptavidin-iminobiotinylated PEI chain bilayer stack deposition at different streptavidin concentrations allowed the determination of the association constant after each bilayer deposition on a single iminobiotinylated photonic sensor. The determined association constants are in good agreement with the values in the literature. These results clearly demonstrate the suitability of reversible bilayer stack deposition for the repeated determination of binding constants on photonic sensors at enhanced sensor signals.

This work was supported by the Austrian NANO Initiative under the PLATON SiNsor grant (project no. 834931).

\section{Notes and references}

1 S. Kurunczi, A. Hainard, K. Juhasz, D. Patko, N. Orgovan, N. Turck, J. Sanchez and R. Horvath, Sens. Actuators, B, 2013, 181, 71-76.

2 (a) J. Satija, V. V. R. Sai and S. Mukherji, J. Mater. Chem., 2011, 21, 14367; (b) H. J. Han, R. M. Kannan, S. Wang, G. Mao, J. P. Kusanovic and R. Romero, Adv. Funct. Mater., 2010, 20, 409-421. 3 (a) S. Suriyanarayanan, H.-H. Lee, B. Liedberg, T. Aastrup and I. A. Nicholls, J. Colloid Interface Sci., 2013, 396, 307-315; (b) Y.-M. Wang, Y. Cui, Z.-Q. Cheng, L.-S. Song, Z.-Y. Wang, B.-H. Han and J.-S. Zhu, Appl. Surf. Sci., 2013, 266, 313.
4 (a) C. E. Hoyle, T. Y. Lee and T. Roper, J. Polym. Sci., Part A: Polym. Chem., 2004, 42, 5301-5338; (b) A. B. Lowe, Polym. Chem., 2010, $1,17$.

5 (a) J. Escorihuela, M. J. Bañuls, R. Puchades and Á. Maquieira, Chem. Commun., 2012, 48, 2116; (b) P. Jonkheijm, D. Weinrich, M. Köhn, H. Engelkamp, P. C. M. Christianen, J. Kuhlmann, J. C. Maan, D. Nüsse, H. Schroeder, R. Wacker, R. Breinbauer, C. M. Niemeyer and H. Waldmann, Angew. Chem., Int. Ed., 2008, $47,4421-4424$

6 (a) C. Hoffmann, K. Schmitt, A. Brandenburg and S. Hartmann, Anal. Bioanal. Chem., 2007, 387, 1921-1932; (b) A. L. Washburn and R. C. Bailey, Analyst, 2010, 136, 227; (c) M.-J. Bañuls, R. Puchades and Á. Maquieira, Anal. Chim. Acta, 2013, 777, 1-16.

7 S. Sam, L. Touahir, J. Salvador Andresa, P. Allongue, J.-N. Chazalviel, A. C. Gouget-Laemmel, C. Henry de Villeneuve, A. Moraillon, F. Ozanam, N. Gabouze and S. Djebbar, Langmuir, 2010, 26, 809-814.

8 P. E. Laibinis, C. D. Bain and G. M. Whitesides, J. Phys. Chem., 1991, 95, 7017.

9 D. Briggs, Surface Analysis of Polymers by XPS and Static SIMS, Cambridge University Press, 2nd edn, 1998.

10 N. M. Green, Adv. Protein Chem., 1975, 29, 85.

11 (a) E. P. Diamandis and T. K. Christopoulos, Clin. Chem., 1991, 37, 625; (b) K. Hofmann, S. W. Wood, C. C. Brinton, J. A. Montibeller and F. M. Finn, Proc. Natl. Acad. Sci. U. S. A., 1980, 77(8), 4666; (c) S. Suna, M. Maa, N. Qiua, X. Huanga, Z. Caia, Q. Huanga and X. $\mathrm{Hu}$, Colloids Surf., B, 2011, 88, 246.

12 N. M. Green, Biochem. J., 1966, 101, 774

13 M. P. Raphael, C. A. Rappole, L. K. Kurihara, J. A. Christodoulides, S. N. Qadri and J. M. Byers, J. Fluoresc., 2011, 21, 647. 